106

Notre Dame Journal of Formal Logic

Volume VII, Number 1, January 1966

\title{
A REMARK ON CONTINUOUS SELECTORS
}

\author{
HENRYK FAST
}

1. For a set $S$ and a class $₹$ of its subsets a function $f: \mathcal{F} \rightarrow S$ with the property that $f(X) \epsilon X$ for $X \epsilon \mathfrak{F}$ is called a selector on $\mathfrak{F}$. If $S$ and $₹$ are topological spaces we can talk about continuous selectors.

We shall restrict ourselves in our considerations to metric spaces only. Let $S$ be a metric space with the metric $d ; \Re$ and $\mathfrak{X}, \mathscr{A} \subset \mathfrak{X}$ be the classes of all arcs and of all continua of $S$. Metrize $X$ using the Hausdorff metric $d_{H}(X, Y)=\inf \left\{r: C_{r}(X) \supset Y, C_{r}(Y) \supset X\right\}, X, Y \subset X .^{1} \quad$ Consider also a stronger metric $d_{H}^{*}(X, Y)=\max \left[d_{H}(X, Y), d_{H}(\partial X, \partial Y)\right]$ on $\mathfrak{X}$.

About the space $S$, make the following assumptions: $S$ in compact, connected, L.C. (locally connected) everywhere and has the property $\left(^{*}\right)$ : A domain $G$ in $S$ is not decomposable by an (compact) arc in $G$ having one point only on its boundary. Notice, that a closed ball for example in the spaces $E_{n}$ and $S_{n}$ (Euclidean and spherical $n$-dimensional) satisfies these conditions. $^{2}$

In the following statement the density is meant in $d_{H}$ metric, the $\left.{ }^{*}\right)$ continuity and the $(*)$-density in $d_{H}^{*}$ metric; $\mathfrak{X}^{*}$ denotes the subclass of $\mathfrak{X}$ consisting of all continua with empty interior.

Proposition: Any selector on $\mathfrak{U}$ (and the more on $\mathfrak{X}$ ) is $(*)$-discontinuous on a set which is dense in $\mathfrak{X}$ and $(*)$-dense in $\mathfrak{X}^{*}$.

This proposition answers the question on the possibility of existence in a local sense of a continuous selector on $\mathfrak{A}$, giving an negative answer to the question asked by Prof. Morton Brown and communicated here by Prof. $\mathrm{K}$. Kuratowski in his recent lecture. The question concerned the existence of a continuous selector on a class of arcs (in $E_{n}$, for instance) with Hausdorff metric.

1. We denote $C_{r}(E)=\{x: \operatorname{dist}(x, E)<r\}, E \subset S$.

2. The property $\left(^{*}\right)$ may be verified here for instance by the application of the "sweep away" theorem [c.f. [1], th. 4 and $4 \mathrm{a}$, p. 350] (as an arc is continuously retractible in itself to a point). 
2. Proof: The proof will be conducted in two steps (a) and (b):

(a) $\mathscr{A}$ is dense in $\mathfrak{X}$. Let $\delta>0$ and let $C$ the component of $C_{\delta}(X)$ containing $X$. $C$ being relatively compact has a $\delta$-net $N=\left\{x_{0}, x_{1}, \ldots x_{m}\right\}: C_{\delta}(N) \supset C$. Due to our supposition about $S, C$ being connected and L.C. is arcwise connected. There exists in $C$ an arc $A$ passing through all the points $x_{0}, x_{1}, \ldots x_{m}$ in this order. This may be established inductively: supposing that such an arc $A_{k}$ exists for the points $x_{0}, x_{1} \ldots x_{k}, k=0,1, \ldots, m-1$ we can extend it to an $A_{k+1}$ due to the property (*) of $S$, (applied to $G=$ $C-A_{k}$; for $k=0$ to $C-\left\{x_{0}\right\}$, which is a domain). From the inclusions $X \subset C \subset C_{\delta}(N) \subset C_{\delta}(A)$ and $A \subset C \subset C_{\delta}(X)$ follows $d_{H}(X, A) \leqslant \delta$ i.e. (a).

(b) Set of points of $(*)$-discontinuity of $f$ is dense in $\mathscr{A}$. Take an arbitrary $X \subset \mathfrak{A}$. We have $x=f(X) \in X$. Let $y \in X, y \neq x$ and let an $\epsilon$ is chosen such that $0<\epsilon<d(x, y)$.

Let $0<\delta<\epsilon$ such that

$$
d(f(Y), x)<\epsilon \text { as } Y \in \mathfrak{A}, d_{H}(Y, X)<\delta .
$$

By (a) there exists an arc $A$ such that $d_{H}(X, A)<\delta$ passing through a net $N=\left\{x_{0}, x_{1}, \ldots x_{2 p}\right\}$ where the additional requirements $y=x_{p}$ and $C_{\delta}\left(N_{i}\right) \supset$ $C, i=1,2$, with $N_{1}=\left\{x_{0}, x_{1}, \ldots x_{p}\right\}, N_{2}=\left\{x_{p+1}, \ldots x_{2 p}\right\}$ clearly may be added.

Let $A$ be represented by the homeomorphism $g:[0,1] \rightarrow S$ of an interval into $S$ and let $g\left(\frac{1}{2}\right)=y$. We have the following relations:

$$
g([t, 1]) \subset A=g([0,1]) \subset C_{\delta}(X) \text { and } X \subset C_{\delta}\left(N_{2}\right) \subset C_{\delta}(g[t, 1])
$$

for $0 \leq t \leq \frac{1}{2}$, which yields

$$
d_{H}(g([t, 1]), X)<\delta, \quad 0 \leq t \leq \frac{1}{2}
$$

whence, putting $z(t)=f(g([t, 1])$ we have by the continuity condition (2.1)

$$
d(z(t), x)<\epsilon, \quad 0 \leq t \leq \frac{1}{2} .
$$

From the definition of selector, $z(t) \epsilon g([t, 1])$. By (2.3) and the choice of $\epsilon, z(t) \neq g\left(\frac{\dot{1}}{2}\right)=y$, hence, $z(t) \epsilon g\left(\left[0, \frac{1}{2}\right)\right)$ or $z(t) \epsilon g\left(\left(\frac{1}{2}, 1\right]\right) . t \rightarrow g([t, 1])$ is a continuous mapping from $[0,1]$ into the space $थ$. Were $f(*)$-continuous in the $\delta$-neighbourhood of $X$, so would be by (2.2) $z(t)$ for $0 \leq t \leq 1$ and, assuming for instance that $z(0) \epsilon: g\left(\left[0, \frac{1}{2}\right]\right)$, we would have $z(t) \epsilon g\left(\left[t, \frac{1}{2}\right]\right)$ for $0 \leq t \leq 1$ and this is impossible, since this implies $\lim _{t \rightarrow 1 / 2} z(t)=y$, which contradicts $(2.3)$. proof.

Since $\left(^{*}\right)$-density of $\mathscr{U}$ in $\mathfrak{X}^{*}$ follows obviously from (a), this ends the

\section{REFERENCE}

[1] C. Kuratowski, Topologie II, Monografie Matematyczne 21, Warszawa 1952. 